\title{
0.9 \% Saline vs $6 \%$ HES 130/0.4 for fluid resuscitation in critically ill patients
}

\author{
Giulia Nenna $\cdot$ Giorgio Costantino
}

Received: 27 February 2013/Accepted: 26 March 2013/Published online: 12 April 2013

(C) SIMI 2013

\section{Background}

The administration of colloids, and hydroxyehtyl starch (HES) in particular, in critically ill patients has been questioned by several studies, with different conclusions regarding outcomes and kidney injury. Older HES solutions have been shown to correlate significantly with acute kidney injury [1], but the question remains open for newer, lower molecular weight and lower MS preparations (tetrastarches). Recently, a Scandinavian trial has reported that $6 \%$ HES 130/0.42 significantly increased mortality in patients with severe sepsis and septic shock [2]. To further assess safety and efficacy of HES solutions, the CHEST investigators have conducted a large-scale trial comparing $6 \%$ HES 130/0.40 (Voluven) to normal saline in ICU patients.

\section{Summary}

Myburgh et al. conducted a prospective, multicentre, blinded, randomized, controlled trial on ICU patients requiring fluid resuscitation therapy at any time and for any reason during their ICU stay.

Enrolled patients were randomly assigned to receive $0.9 \%$ saline (saline group) or $6 \%$ HES 130/0.4 (HES group) during the ICU stay.

Fluid resuscitation therapy was defined as the administration of a bolus of IV fluid over and above that required for maintenance and replacement fluids and the need for its administration was determined by the treating physician

G. Nenna $(\bowtie) \cdot$ G. Costantino

Arese, MI, Italy

e-mail: giu.nenna@gmail.com but had to be supported by at least one suggestive clinical sign. Exclusion criteria were the presence of any contraindication to either saline or HES (known allergy to HES, intracranial hemorrhage, renal impairment, severe hypenatremia/hyperchloremia, women of childbearing age or breastfeeding, previous heart or liver transplantation surgery, patients admitted for burns), imminent death or unadvisable aggressive treatment.

The primary endpoint was all-cause mortality in the enrolled population at 90 days. The study was designed to detect a difference in mortality of at least $3.5 \%$ with a power of $90 \%$ assuming a mortality rate of $26 \%$. The primary outcome was also examined in six pre-specified subgroup pairs (presence of AKI at baseline, diagnosis of sepsis on admittance, presence of trauma, APACHE score $>$ or $<25$, receipt of HES before randomization) to detect possible influence of the study fluid in certain specific conditions as previous studies have suggested $[5,6]$.

Secondary outcomes were incidence of acute kidney injury assessed through RIFLE criteria, use of renalreplacement therapy (RRT), new organ failure assessed through a SOFA score of 3 or more for any system, duration of mechanical ventilation and RRT, cause-specific mortality.

The results did not show a significant difference in any of the analyses conducted with the exception of a higher rate of renal replacement therapy in patients treated with HES vs saline ( 7 vs $5.8 \%, p=0.04$ ).

\section{Strengths of the study}

- A large population of patients was enrolled;

- it addresses a topic that is presently debated and is relevant both to patient outcome and to cost management; 
- the study was well designed, the study protocol and statistical analysis plan were published before the end of the study[3,4], subgroups were pre-specified and post hoc analyses were stated and motivated;

- the study was modeled after SAFE trial (making it easier to merge the two studies for future metaanalyses).

\section{Weaknesses of the study}

- As the authors themselves state in the article, mortality rates (17-18\%) were lower than expected: this might mean that the inclusion criteria used did not select the expected population for the study.

\section{Question marks}

- Mortality rate was low especially if compared to other studies recruiting patients in the ICU, where higher mortality rates are reported [5, 6]. Patients also had a quite high PVC at baseline on average and required relatively small quantities of the study fluid for fluid resuscitation therapy (about $1 \mathrm{~L}$ on the first day): this might mean that the population enrolled was less critically ill than the "standard" ICU patients and might affect the external validity of the study;

- it would be interesting to know the characteristics of the patients at the beginning of renal replacement therapy for the two groups to assess if the HES group was in any way different from the other, since the need for renal replacement therapy, although blind, was left to clinical judgment.

\section{Clinical bottom line}

HES does not appear to be superior to saline in both safety and efficacy outcomes and might actually be worse for the renal outcome: physicians should continue using crystalloids as default resuscitation fluids unless otherwise suggested by the clinical judgment in selected cases.

Conflict of interest None.

\section{References}

1. Brunkhorst FM, Engel C, Bloos F et al (2008) Intensive insulin therapy and pentastarch resuscitation in severe sepsis. N Engl $\mathrm{J}$ Med 358:125-139

2. Perner A, Haase N, Guttormsen AB, et al (2012) Hydroxyethyl starch 130/0.42 versus Ringer's acetate in severe sepsis. N Engl J Med 367:124-134 [(2012) Erratum. N Engl J Med 367:481]

3. Crystalloid versus Hydroxyethyl Starch Trial (CHEST) Management Committee (2011) The Crystalloid versus Hydroxyethyl Starch Trial: protocol for a multi-centre randomized controlled trial of fluid resuscitation with $6 \%$ hydroxyethyl starch (130/0.4) compared to $0.9 \%$ sodium chloride (saline) in intensive care patients on mortality. Intensive Care Med 37:816-823

4. Myburgh J, Li Q, Heritier S, Dan A, Glass P (2012) Statistical analysis plan for the Crystalloid Versus Hydroxyethyl Starch Trial (CHEST). Crit Care Resusc 14:44-52 [(2012) Erratum. Crit Care Resusc 14:164.]

5. Guidet B, Martinet O, Boulain T et al (2012) Assessment of hemodynamic efficacy and safety of $6 \%$ hydroxyethylstarch $130 / 0.4$ vs. $0.9 \% \mathrm{NaCl}$ fluid replacement in patients with severe sepsis: the CRYSTMAS study. Crit Care 16(3):R94

6. James MF, Michell WL, Joubert IA, Nicol AJ, Navsaria PH, Gillespie RS (2011) Resuscitation with hydroxyethyl starch improves renal function and lactate clearance in penetrating trauma in a randomized controlled study: the FIRST trial (fluids in resuscitation of severe trauma). Br J Anaesth 107:693-702 